OPEN ACCESS

Edited by:

Wanwipa Vongsangnak,

Kasetsart University, Thailand

Reviewed by: Rúbia Carvalho Gomes Corrêa, Cesumar University -

UniCesumar, Brazil

Yanchun Shao,

Huazhong Agricultural

University, China

${ }^{*}$ Correspondence:

$\mathrm{Bin} \mathrm{He}$

hebin.li@foxmail.com

Specialty section:

This article was submitted to

Food Chemistry,

a section of the journal

Frontiers in Nutrition

Received: 12 October 2021 Accepted: 24 November 2021 Published: 23 December 2021

Citation:

Lv G, Zhu Y, Cheng X, Cao Y, Zeng B, Liu X and He B (2021) Transcriptomic Responses of Cordyceps militaris to Salt Treatment During Cordycepins

Production. Front. Nutr. 8:793795. doi: 10.3389/fnut.2021.793795

\section{Transcriptomic Responses of Cordyceps militaris to Salt Treatment During Cordycepins Production}

\author{
Gongbo Lv ${ }^{1}$, Yue Zhu ${ }^{1}$, Xiaojie Cheng ${ }^{2}$, Yan $\mathrm{Cao}^{3}$, Bin Zeng ${ }^{1,4}$, Xinping $\mathrm{Liu}^{1}$ and Bin $\mathrm{He}^{1 *}$ \\ 1 Jiangxi Key Laboratory of Bioprocess Engineering and Co-innovation Center for in-vitro Diagnostic Reagents and Devices of \\ Jiangxi Province, College of Life Sciences, Jiangxi Science and Technology Normal University, Nanchang, China, ${ }^{2}$ College of \\ Life Sciences, Sichuan Normal University, Chengdu, China, ${ }^{3}$ Information Institute of Sichuan Academy of Agricultural \\ Sciences, Chengdu, China, ${ }^{4}$ College of Pharmacy, Shenzhen Technology University, Shenzhen, China
}

Cordycepin is a major bioactive compound found in Cordyceps militaris (C. militaris) that exhibits a broad spectrum of biological activities. Hence, it is potentially a bioactive ingredient of pharmaceutical and cosmetic products. However, overexploitation and low productivity of natural C. militaris is a barrier to commercialization, which leads to insufficient supply to meet its existing market demands. In this study, a preliminary study of distinct concentrations of salt treatments toward C. militaris was conducted. Although the growth of $C$. militaris was inhibited by different salt treatments, the cordycepin production increased significantly accompanied by the increment of salt concentration. Among them, the content of cordycepin in the $7 \%$ salt-treated group was five-fold higher than that of the control group. Further transcriptome analysis of samples with four salt concentrations, coupled with Gene Ontology (GO) analysis and Kyoto Encyclopedia of Genes and Genomes (KEGG) pathway enrichment, several differentially expressed genes (DEGs) were found. Finally, dynamic changes of the expression patterns of four genes involved in the cordycepin biosynthesis pathway were observed by the quantitative real-time PCR. Taken together, our study provides a global transcriptome characterization of the salt treatment adaptation process in C. militaris and facilitates the construction of industrial strains with a high cordycepin production and salt tolerance.

Keywords: cordycepin, Cordyceps militaris, salt treatment, molecular mechanism, transcriptome

\section{INTRODUCTION}

Cordyceps militaris (C. militaris), or known as North Cordyceps sinensis, is an entomogenous fungus belonging to the Ascomycota, Hypocreales, and Ergotaceae, as spores are produced internally inside a sac, called ascus (1). It is a well-known edible and medicinal fungus and one of the most important traditional Chinese medicines. Contrary to Cordyceps sinensis (DongChongXiaCao) in Chinese herbs, which is unique to China, C. militaris is a worldwide species and is also distributed in almost all provinces and regions in China. Its soporophore can parasitize larvae or grow on the pupae of lepidopteran insects. These insects are located on the scales half-buried on the forest floor or under the layer of deciduous branches from spring to autumn (2). C. militaris has been extensively used as a folk medicine in areas of East Asia for the revitalization of various systems of the body from ancient times, and currently it is also widely used in Western countries (3). Since C. militaris produces a variety of bioactive compounds with functional properties, it has long been utilized as a 
dietary supplement (tonic food) or herbal medicine for the pharmaceutical and cosmetics industries for many years $(4,5)$. Various bioactive metabolites isolated from $C$. militaris, such as cordycepin ( $3^{\prime}$-deoxyadenosine) (6), cordycepic acid, carotenoid, ergosterol, and cordyceps polysaccharide (7), have been explored extensively for extending its applications; particularly, several of them are extracted and made into tablets and capsules (8). In addition, fermentation and downstream processes or other bioprocess developments have permitted a prospect in the production of these specific bioactive metabolites as functional ingredients for diversified applications (9).

Among the aforementioned bioactive metabolites, the cordycepin ( $3^{\prime}$-deoxyadenosine) has attracted the most attention in C. militaris, and its potential therapeutic effect has been mostly mentioned and investigated for clinical trials against cancer (10). As the main bioactive ingredient of $C$. militaris, cordycepin is used as a transcription inhibitor for its lack of hydroxyl moiety at the C3 position. When cordycepin is integrated into the RNA chain, it will lead to the termination of transcription (11). Moreover, it has been reported that cordycepin can inhibit cell proliferation and induce cell apoptosis via binding signaling molecules, which resulted in anti-inflammatory action (12-14). Cordycepin and adenosine have a similar structure, except for the lack of a $3^{\prime}$ hydroxyl group on cordycepin. Even with such a tiny difference, cordycepin and adenosine exhibit completely distinct biological activity, and cordycepin is reported to interfere with many molecular and cellular processes within cells. Furthermore, extensive research results showed that cordycepin has many other diversified biological activities, such as a very potent anticancer, anti-ischemic, and anti-oxidant protective effect. Exactly, cordycepin binds the $\mathrm{A} 3$ adenosine receptor, which results in activating $\mathrm{G}$ protein (inhibitory regulative guanine nucleotidebinding protein). Afterward, the cAMP formation was inhibited, then the serine/threonine kinase glycogen synthase kinase (GSK)-3 $\beta / \beta$-catenin signaling pathway and subsequently cell division were indirectly inactivated and suppressed, respectively (15). Additionally, it has been also documented that cordycepin effects on inhibiting platelet aggregation, inducing steroid formation, and with broad-spectrum antibiotic activity by inhibiting $\mathrm{NAD}^{+}$-dependent DNA ligase $(13,16,17)$.

With over seven-decade-long historical investigations of cordycepin, it was first isolated from C. militaris in 1950 (6), and the chemical structure of cordycepin was confirmed to be $3^{\prime}$-deoxyadenosine via mass spectrometry data, infrared spectroscopy, and nuclear magnetic resonance spectroscopy data, combined with the characteristics of the ultraviolet absorption spectrum (18, 19). Meanwhile, the cordycepin biosynthetic pathway has been exploited for over half-centuries. Though several hypotheses concerning cordycepin biosynthetic pathways were proposed, clear experimental evidence to clarify "how cordycepin is produced" was lacking. With the completion of the whole genome sequencing of $C$. militaris, Xia et al. first identified the gene cluster responsible for the cordycepin biosynthesis, and the cordycepin biosynthesis pathway was further fulfilled in 2017 (20, 21). There were four genes (Cns1-Cns4) physically linked as the cordycepin biosynthetic gene cluster and encoded proteins with distinct conserved domains, which differently mediated cordycepin metabolism. Among them, Cns1 (CCM_04436) contains the oxidoreductase/dehydrogenase domain, and Cns2 (CCM_04437) possesses the HDc family of metal-dependent phosphohydrolase domain. There were two functional domains contained in Cns3 (CCM_04438): an $\mathrm{N}$-terminal nucleoside/nucleotide kinase (NK) and a C-terminal HisG family of ATP phosphoribosyltransferases. Lastly, Cns4 (CCM_04439) was identified as a member of the putative pleiotropic drug resistance (PDR) family of ATP-binding cassette (ABC) transporters (20). According to the finding of Xia et al., cordycepin biosynthesis begins at adenosine, and the hydroxyl phosphorylation is catalyzed by $C n s 3$ to produce adenosine- $3^{\prime}$-monophosphate (3' AMP). Subsequently, $3^{\prime}$ AMP was dephosphorylated to $2^{\prime}$-carbonyl-3'-deoxyadenosine $\left(2^{\prime}-\right.$ $\left.\mathrm{C}-3^{\prime}-\mathrm{dA}\right)$ by phosphohydrolase activity of Cns2 (21). Finally, the $2^{\prime}-\mathrm{C}-3^{\prime}-\mathrm{dA}$ was converted to cordycepin via oxidoreduction reactions mediated by Cns1. Simultaneously, it was noteworthy that pentostatin (PTN, 2'-deoxycoformycin), an irreversible inhibitor of adenosine deaminase, was yielded in coupling with cordycepin by phosphoribosyltransferase domain of Cns3 (22). Consequently, this process inhibited the deamination of cordycepin to $3^{\prime}$-deoxyinosine to maintain the stability of cellular cordycepin through the bacterium protector-protégé strategy (23). Furthermore, when cordycepin accumulation reaches a high (toxic) intracellular level, such PTN will be pumped out of the cell by the Cns4 transporter for neutralizing cordycepin to nontoxic $3^{\prime}$-deoxyinosine (24).

To meet the existing and increasing market demand of cordycepin production, strategies for the enhancement of this medical component production in Cordyceps spp. are warranted. Plentiful literature has been reported on diversified approaches which focused on the improvement of cordycepin content, such as C. militaris strain mutagenesis, optimization of medium composition, culture conditions, and extraction methods. Among them, optimization of medium composition is usually the first choice to increase the content of cordycepin. Growth supplements and additives, such as sugar, amino acids (L-alanine, glycine, casein hydrolysate, and glutamine), vitamins, inorganic salts (ferrous sulfate and sodium selenite), nucleoside analog (hypoxanthine and adenosine), porcine liver extracts, or vegetable oils (peanut and cottonseed oil), were proved to be successful $(8,25-30)$. Moreover, ways of genetic and metabolic engineering are noteworthy, and two main methods are mentioned as follows, the overexpression and disruption of the gene on the cordycepin biosynthetic cluster. It has been reported that the production of cordycepin was increased by $25 \%$ by deletion of Cns3 when compared with the control (21). Meanwhile, overexpression of the single Cns1, Cns2, and Cns3 along with the Cns1/Cns2 fusion gene was also performed via individually transformed the control strain of C. militaris. It was observed that in contrast to the wildtype strain, the yield of cordycepin in the transformant was engineered with the Cns1/Cns2 fusion gene that was increased 2.7-fold, whereas no obvious differences were found between the control and transformants engineered with single genes. 
Moreover, previous reports showed that cordycepin production could be increased by ultraviolet, ${ }^{60} \mathrm{Co} \gamma$-ray, and blue light LED irradiation. For instance, the cordycepin titer of Paecilomyces hepiali ZJB18001strain was increased 2.3 -fold via ${ }^{60} \mathrm{Co} \gamma$-ray and ultraviolet irradiation compared with that from the wild strain $(27,29,31)$. Additionally, nutrient stress (distinct carbon, nitrogen, phosphorous sources, and ratios) and environmental stress (lighting time, temperature, $\mathrm{pH}$, and shaking speed) were also effective strategies. It was reported that glucose and casein hydrolysate were the most beneficial carbon and nitrogen sources in cordycepin production (2.3-fold improvement relative to that of the control), and especially, production was greatly affected by casein hydrolysate (29).

Salt stress has negative effects on the growth and survival of organisms, such as leading to water loss and cell shrinking, and several metabolic changes are observed $(32,33)$. However, to the best of our knowledge, a detailed mechanism of the response of $C$. militaris to salt treatment and its effect on the enrichment of cordycepin remains to be elucidated. The high-salt fermenting environment prolongs the process of substrate catabolism and biosynthesis for the decrease in cell growth and enzyme performance. Thus, the construction of industrial strains with a high cordycepin production and salt tolerance for industrial production is highly desirable. To better comprehend the molecular mechanism of salt-action on cordycepin production, a transcriptome analysis was carried out in the present study. Our study demonstrated the effect of different salt concentrations on cordycepin contents in the CM01 strain, followed by a transcriptome analysis based on RNA sequencing of each salt-treated C. militaris, coupled with gene oncology (GO) analysis and Kyoto Encyclopedia of Genes and Genomes (KEGG) pathway enrichment of differentially expressed genes (DEGs). Generally, there is an array of genes that regulates the synthesis of bioactive metabolites, and these genes were upregulated or downregulated during the biosynthesis of cordycepin. Therefore, mRNA expression analysis of several genes involved in the cordycepin biosynthesis pathway was also performed to determine the effect of different salt treatments on potential target genes whose modulation may increase cordycepin production to meet the industrial needs.

\section{MATERIALS AND METHODS \\ Fungal Strain and Cultivation}

In this study, the C. militaris strains involved in the experiments was the commonly used strain in our laboratory, CM01strain. This strain is an isolate of $C$. militaris with a mating type of M4T1-1. A basal medium, containing (g/L) glucose 20, peptone 5, $\mathrm{KH}_{2} \mathrm{PO}_{4} 1, \mathrm{MgSO}_{4} \cdot 7 \mathrm{H}_{2} \mathrm{O} 0.5$, Vitamin $\mathrm{B}_{1} 0.05$, and Vitamin $\mathrm{B}_{2}$ 0.05 , was used. In total, $200 \mathrm{~g}$ potato was milled and boiled with water and filtrated with gauze. The filtrates were mixed with the basal medium, and then water was added to the final volume of $1,000 \mathrm{ml}$ to prepare the liquid medium. After that, the $\mathrm{pH}$ of the liquid medium was adjusted to 7.2. The prepared liquid medium was divided into $250 \mathrm{ml}$ flasks ( $100 \mathrm{ml}$ per flask), coupled with $2 \mathrm{~g}$ agar, and one of them was not added agar for strain activating. In total, $0,3,5$, and $7 \mathrm{~g} \mathrm{NaCl}$ per $100 \mathrm{ml}$ were added to the medium as treatments, which were equivalent to control, slight stress, moderate stress, and severe salt treatment, respectively. And then all of the media were sterilized in moist heat sterilization autoclave for $20 \mathrm{~min}$ at $121^{\circ} \mathrm{C}$. When the culture medium was cooled, C. militaris strain CM01 was incubated in the prepared liquid medium for activating strain under aseptic conditions. The vibration culture with a speed of $120-140 \mathrm{rpm} / \mathrm{min}$ was employed to expand the culture when the white mycelium grows from the seed block (generally $24 \mathrm{~h}$ ). The activated strain was followed by inoculation on distinct salt-treated potato dextrose agar (PDA) plates and cultured in darkness at a temperature of $22^{\circ} \mathrm{C}$. The culture was incubated for 5-7 days. After the media were covered by mycelia, these plates were transferred to the light culture at a temperature of $22^{\circ} \mathrm{C}$ and lighting intensity of $600 \mathrm{~lx}$ for $15 \mathrm{~h}$ each day (about 5 days). It was followed by scraping and then drying the C. militaris mycelia overnight at $60^{\circ} \mathrm{C}$ for the biomass determination. All experiments were performed in triplicate to ensure reproducibility.

\section{Extraction of Cordycepin From C. militaris}

After 5 days of light culture, C. militaris mycelium was harvested, and then cordycepin content was determined for the next analysis. Specifically, the mycelia was lyophilized, vacuum freezedried to a constant weight, and ground to powder. Two grams of dry powder were weighted from each sample to perform High Performance Liquid Chromatography (HPLC) detection. HPLC assay was conducted on Waters Alliance e2695 HPLC (Milford, MA, USA) using a UV detector set at $260 \mathrm{~nm}$ equipped with a ZorbaxSB-C18 column $(4.6 \times 250 \mathrm{~mm}, 5 \mu \mathrm{m})$. The analysis conditions were as follows: mobile phase, $85 \%$ ultrapure water/methanol $(85: 15, \mathrm{v} / \mathrm{v})$, and the elution rate was $1.5 \mathrm{ml}$ $\mathrm{min}^{-1}$; injection volume, $20 \mu \mathrm{l}$. A standard cordycepin curve was generated using $0.02-0.25 \mu \mathrm{g} / \mathrm{ml}$ cordycepin standard (SigmaAldrich, Burlington, MA, USA). The cordycepin yield was calculated using the detected peak area according to the standard curve. The cordycepin concentration of mycelia presented in our study was calculated by normalizing in the equal biomass.

\section{Extraction of Total RNA, Library Establishment, and Transcriptome Sequencing}

Total RNA extraction was performed with a fungal RNA kit (Omega Bio-tek, Norcross, GA, USA) by following the operating instructions, coupled with DNA digestion. NanoDrop ND1000 spectrophotometer (Thermo Scientific, Wilmington, DE, USA) and Bioanalyzer 2100 (Agilent Technologies, Palo Alto, CA, USA) were employed to analyze RNA concentration and integrity. Thereafter, mRNA was enriched by Oligo (dT) beads. The mRNAs were fragmented and used as templates to synthesize cDNA. The cDNA fragments were purified using QiaQuick PCR extraction kit, end-repaired, single-nucleotide adenine addition, and ligated to Illumina sequencing adapters to create the cDNA library. The size of the ligation products was selected by agarose gel electrophoresis, amplified, and sequenced using Illumina HiSeqTM2500 (Biomarker Biotechnology Co., Beijing, China) (34). RNA-Seq data of C. militaris under salt treatment were 
deposited in the NCBI/SRA database (https://www.ncbi.nlm.nih. gov/sra), under the Bioproject accession number PRJNA770191; BioSample: SAMN22211876-SAMN22211879.

\section{Transcriptome Analysis}

The transcriptome datasets are raw reads containing adapters or low-quality bases. Therefore, reads will be further filtered by our previous criterion to get clean reads and thus removed (35). Moreover, Bowtie2 software (http://bowtie-bio.sourceforge. net/bowtie2) was performed to remove reads that mapped to ribosome RNA (rRNA) database to get the final clean reads, which were further employed for assembly and transcriptome analysis (36). For gene expression analysis, the obtained cleanread data sets of the four different cultures were mapped to the reference genome C. militaris CM01 (NCBI accession number. AEVU00000000) using Tophats2 (v2.0.3.12) (37). RSEM software was used to quantify gene abundances, and the quantification of gene expression level was normalized using the Fragments Per Kilobase of transcript per Million mapped reads (FPKM) method $(38,39)$.

\section{DEGs Annotation}

Differentially expressed genes across samples were identified using edge $\mathrm{R}$ on the $\mathrm{R}$ package (version 3.4.2) according to the RSEM results (40). The absolute value of fold change $\geq 2$ and false discovery rate (FDR) within 0.05 were set as the threshold for the detection of significant DEGs among four treatments (CK vs. NaCl-3, -5, and -7) (34). Then the identified DEGs were carried out into hierarchical clustering, with the KEGG pathway enrichment analysis. To further analyze the DEG annotations, GO functional classification and Kyoto Encyclopedia of Genes and Genomes (KEGG) pathway enrichment analysis were carried out based on the GO database (41) and KEGG database (42).

\section{Quantitative Real-Time PCR Validations of DEGs}

To validate the transcriptional level results from RNA-Seq data analysis, four genes (encode cordycepin synthetase) Cns1, Cns2, Cns3, and Cns4 are involved in the cordycepin biosynthesis in C. militaris were selected for real-time RT-PCR validation. The qRT-PCR template cDNA was synthesized from $0.5 \mu \mathrm{g}$ of total RNA by Fungal RNA Kit. Reverse transcription of each RNA sample was performed to get the first-strand cDNA using the PrimeScript RT reagent Kit with gDNA Eraser (Takara, Dalian, China). Each qRT-PCR reaction system had a total volume of $10 \mu \mathrm{l}$, containing cDNA, relevant primers, and the SYBR Green Real-time PCR Master Mix (Takara). Real-time RT-PCR was performed using Real-Time PCR System (Bio-Rad, Hercules, CA, USA). GAPDH was served as the internal control (reference gene) for normalization of the target gene expression and to correct for variation between samples. The thermal cycle for RTPCR was as follows: $95^{\circ} \mathrm{C}$ for $2 \mathrm{~min}$, followed by 40 cycles of $95^{\circ} \mathrm{C}$ for $10 \mathrm{sec}, 60^{\circ} \mathrm{C}$ for $15 \mathrm{sec}$, and $72^{\circ} \mathrm{C}$ for $20 \mathrm{sec}$. Melting curve analyses of the amplification products were performed at the end of each PCR reaction to ensure that only specific products were amplified. Primers used for the candidate genes are designed according to Illumina sequencing data by using

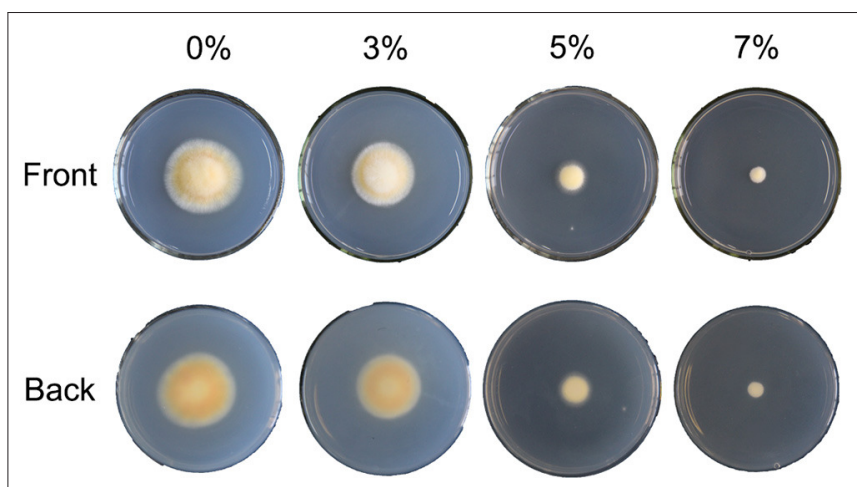

FIGURE 1 | The phenotype of $C$. militaris is affected by different salt treatments. $0,3,5$, and $7 \%$ are equivalent to the control, slight, moderate, and severe salt treatment, respectively.

Primer Premier 5 and listed in Supplementary Table S1. The comparative $2^{-\Delta \Delta C T}$ method was employed to calculate relative expression levels between the target genes.

\section{Data Analysis}

Three independent experiments were performed, and all the data in this study are presented as mean \pm SE of three replicates. Data from the same period were evaluated by one-way nested ANOVA, followed by the least significant difference test (LSD) for mean comparison. One tail student $t$-test was conducted between the control and salt-treated groups to calculate the $p$-values. All statistical analysis was performed with SAS 9.20 software (SAS Institute Inc., Cary, NC, USA) at the $p<0.05$.

\section{RESULTS}

\section{Growth Characteristics and Cordycepin Production From C. militaris After Salt Treatments}

To explore the effects of salt treatment on cell growth and cordycepin production of C. militaris, a preliminary study of distinct concentrations of salt treatments was conducted. After strain activation, incubation, and dark and light culture, the phenotypes of C. militaris mycelioid colonies of the salttreated group were significantly different from the control (Figure 1). As the result showed, the growth of $C$. militaris was significantly restricted by salt treatment and the colony size descends remarkably as the salt concentration increases, especially under the 5 and $7 \%$ salt treatments. When the salt concentration is beyond $7 \%$, the mycelia of C. militaris cannot even grow at all. It was followed by determining the biomass of the C. militaris mycelia. According to the obtained results, the biomass was decreased with the increase of salt concentration (Supplementary Figure S1). Analogous to colony diameter, the lowest biomass of the C. militaris mycelia was found in the $7 \%$ salt-treated group.

High Performance Liquid Chromatography detection assay was performed to determine the production of cordycepin of 


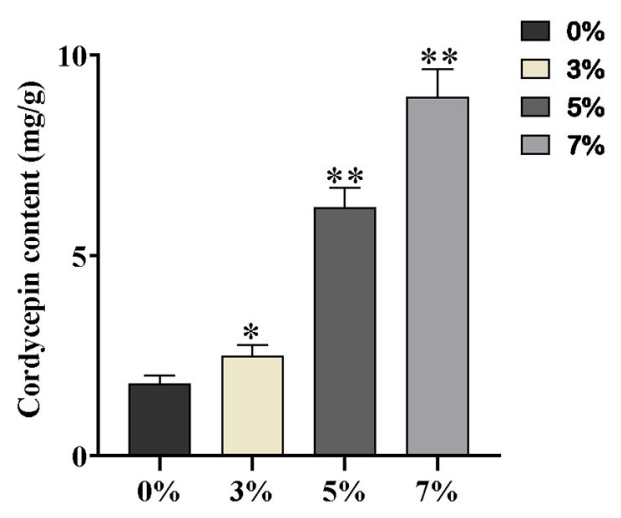

FIGURE 2 | The contents of cordycepin with $\mathrm{mg} / \mathrm{g}$ dry weight 0, 3, 5, and 7\% are equivalent to the control, slight, moderate, and severe salt treatment, respectively. Asterisks indicate statistically significant differences between groups (Student's t-test): * and ** indicating significance level was accepted at $P \leq 0.05$ and $P<0.01$, respectively, as compared to control.

each salt-treated group. Although the growth of C. militaris was inhibited by distinct salt treatments, the cordycepin production of per unit volume of mycelium has increased notably, accompanied by the improvement of salt concentration (Supplementary Figure S2). Noteworthily, the content of cordycepin treated with $7 \%$ salt was five-fold higher than that of the control group, and the remaining cordycepin content with $\mathrm{mg} / \mathrm{g}$ dry weight is presented in Figure 2.

\section{Transcriptome Overview}

To investigate the molecular mechanism of salt treatment that increases the per-volume content of cordycepin in C. militaris, a transcriptome analysis based on RNA sequencing of each salttreated C. militaris was performed. Four cDNA samples from $C$. militaris mycelia were processed by the Illumina HiSeq platform, and the transcriptome data obtained by sequencing results were listed in Table 1. This resulted in the generation of 40.81, 40.50, 45.22 , and 55.58 million clean reads per library, respectively. The GC content for all treatments was $\sim 57 \%$, and the $\% \geq$ Q30 (99.9\% accuracy of bases) was $>94 \%$ for all samples, indicating a good quality of the sequencing data which can be used to do the following analysis. After mapping the sequenced reads to the reference genome, more than $92.5 \%$ of the reads in all four samples were mapped. Additionally, a total of 134,436, 155,697, 159,689 , and 162,615 single nucleotide polymorphism (SNP) numbers were generated, and most of them were synonymous mutations according to the subsequent annotation.

\section{DEGs Analysis}

To identify genes with altered expression levels with salt treatment, the overall transcription levels of genes were quantified by Revenue Passenger Kilometers (RPKM) metrics. According to global transcriptional changes from normalizing the DEG data (Supplementary Table S2), a total of 3,885 genes showed altered expression levels in the three salinity treatment groups, as compared to the control. There were 2,917 DEGs in the
TABLE 1 | Summary of the sequencing data of C. militaris under different salt concentrations.

\begin{tabular}{lcccc}
\hline Samples & Control & NaCl-3 & NaCl-5 & NaCl-7 \\
\hline Clean reads & 40817724 & 45502268 & 45225634 & 55588246 \\
GC Content & $57.51 \%$ & $57.26 \%$ & $57.30 \%$ & $57.57 \%$ \\
Q30 & $94.47 \%$ & $95.00 \%$ & $94.28 \%$ & $94.74 \%$ \\
Mapping rates & $92.52 \%$ & $92.71 \%$ & $93.01 \%$ & $93.79 \%$ \\
SNP number & 134436 & 155697 & 159689 & 162615 \\
Genic SNP & 102703 & 112702 & 115509 & 116064 \\
Intergenic SNP & 31733 & 42995 & 44180 & 46551 \\
\hline
\end{tabular}

Control vs. NaCl-7 group, most of all groups, 1,501 upregulated genes (51\%) and 1,416 downregulated genes (49\%); followed by 2,413 and 2,371 DEGs in the Control vs. NaCl-5 and Control vs. NaCl-3 groups, separately. In addition, there were no significant differences between NaCl-3 vs. NaCl-5 and NaCl-5 vs. NaCl-7 groups (Figure 3A). Venn diagram analysis of DEGs between the control and salt treatment treatments revealed that 1,396 DEGs were commonly shared among the distinct salt treatments, the other commonly shared DEGs between every two groups were 180, 281, and 563, respectively (Figure 3B). Moreover, a heatmap was drawn to present the hierarchical clustering of the DEGs among samples (Supplementary Figure S3). As shown in the heatmap, the expression profiles between the control and three salt-treated groups were different, illustrating that the effect of different salt concentrations on C. militaris is distinct GO functional classification of DEGs.

To analyze the function of DEGs and gene annotation on the control and salt treatment strains, GO classification and functional enrichment were performed. Generally, GO annotation consists of three types: biological process, cellular component, and molecular function. Based on GO annotation, a total of 1,096, 1,060, and 866 DEGs were assigned to the ontologies "biological process", "cellular component", and "molecular function", respectively; for the biological process classification, most of DEGs focused on "metabolic process", "cellular process", and "single-organism process" item, etc. In the part of cellular component, "cell", "membrane", and "membrane part" were the most highly represented categories; while for the molecular function classification, "catalytic activity" was the most enriched GO term, followed by "binding", and "transporter activity", and the detailed distribution of GO terms are illustrated in Figure 4.

\section{KEGG Pathway Enrichment of DEGs}

To obtain a better insight into the interactions of functional genes by pathway-based analysis, all the genes were mapped to the KEGG database to classify the biological functions of the DEGs. Specific gene enrichment was observed in 79 pathways. Nineteen pathways showed DEG enrichment (Figure 5). It was observed that the pathway with the lowest $q$ value and the largest number of DEGs was the pathway related to amino sugar and nucleotide sugar metabolism, followed by the pathways related to the synthesis of unsaturated fatty acids, 

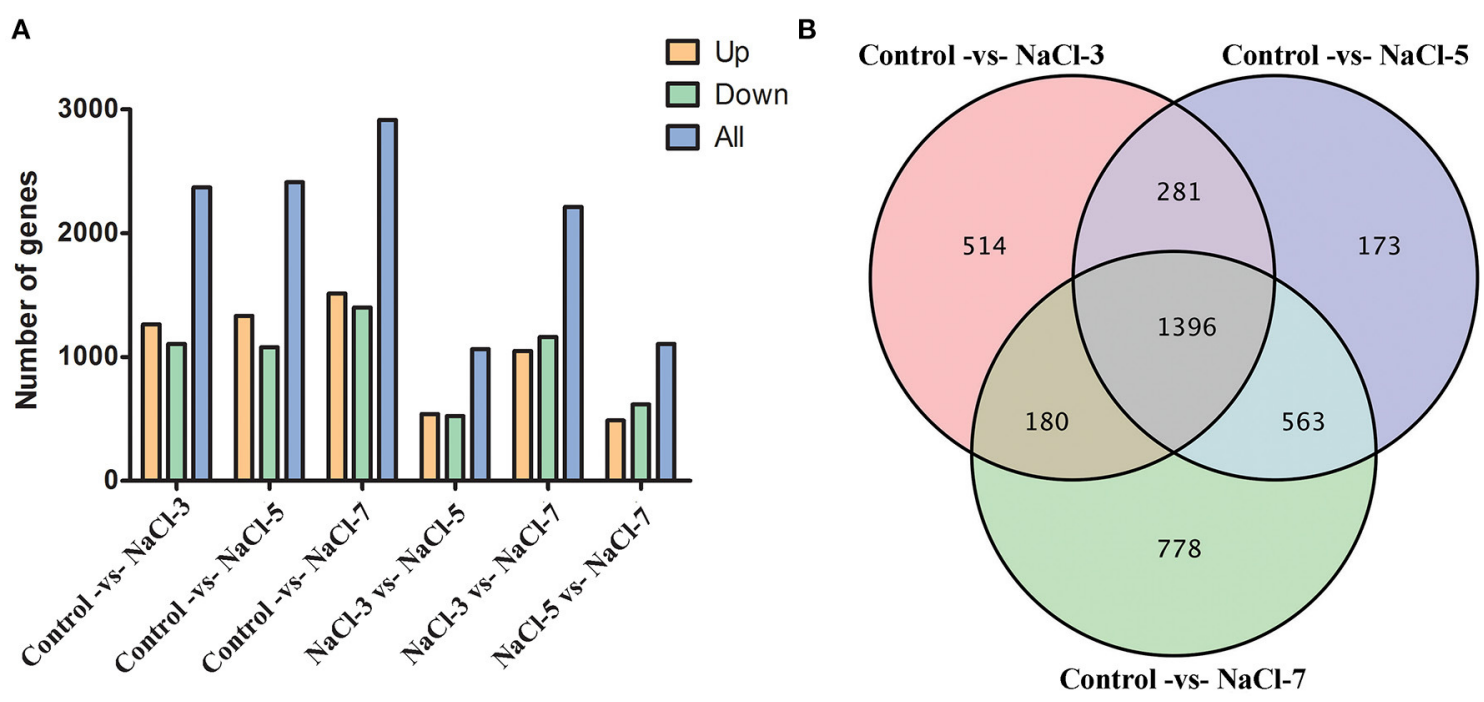

FIGURE 3 | Distribution of differentially expressed genes (DEGs) in the six samples. (A) DEGs' distribution between control and three different salt treatments; (B) Venn diagram of the commonly expressed genes between samples. $\mathrm{NaCl}-3,5$, and 7 represent 3, 5, and 7\% salt concentrations, respectively.

nitrogen metabolism, and glycerolipid metabolism. Furthermore, there were 9 pathways with a $P$-value $<0.05$, such as "fatty acid degradation" (Supplementary Table S3). "Amino sugar and nucleotide sugar metabolism", "Biosynthesis of unsaturated fatty acids", and "Glycerolipid metabolism" showed an even greater significant enrichment $(P<0.01)$.

\section{Analysis of the Genes Related to Cordycepin Synthesis Pathway}

Since the cordycepin content was significantly improved by salt treatment, DEGs related to cordycepin synthesis were carefully identified by KEGG pathway analysis. According to the previously reported and generally accepted cordycepin biosynthesis pathway, this pathway started from adenosine to synthesize cordycepin and PTN (Figure 6). Four key genes involved in the cordycepin synthesis pathway, Cns1, Cns2, Cns3, and $C n s 4$, were performed qRT-PCR validation. Though the fold changes of these genes in qRT-PCR were slightly different from that of RNA-Seq results, they shared a similar change tendency (Supplementary Figure S4). Based on the analysis of the cordycepin synthesis pathway and PCR results, the expression levels of Cns1 and Cns2 were decreased with the increase of salt concentration compared with the control, except Cns1 on NaCl7 group and $\mathrm{Cns} 2$ on $\mathrm{NaCl}-3$ group. Cns 1 and $\mathrm{Cns} 2$ showed high expression levels on these two groups beyond that of the control. The dynamic changes of Cns 1 and Cns 2 expression levels illustrated that the response of these two genes to distinct salt treatments may differ. On the contrary, the expression level of Cns3 was upregulated compared to the control, especially in the NaCl-7 group. This result indicated that the pathway of adenosine into 3 AMP might be greatly activated and finally increase the content of cordycepin under the treatment of $7 \%$ salt concentration. Additionally, the expression level of Cns 4 was also increased remarkably with the increment of salt concentration, revealing that salt treatment might likely facilitate the out pumping of PTN.

\section{DISCUSSION}

Considerable efforts have been put into the improvement of cordycepin production. The previous report showed that cordycepin content could be increased by nutrient stress (distinct carbon, nitrogen, phosphorous sources, and ratios), environmental stress (lighting time, temperature, $\mathrm{pH}$, and shaking speed), and adding supplementation (inorganic salts, porcine liver extracts or vegetable oils, amino acids, or nucleoside analog) (8, 27-29). Though the pathway and related biosynthesis mechanisms of cordycepin have been well documented, the dependency of $C$. militaris activity on different salt concentrations in a medium remains unclear. To obtain more insight on C. militaris adaptations to salt stress conditions, precise physiological knowledge is needed. Therefore, a preexperiment was carried out to evaluate the effect of salinity treatment on cordycepin production in C. militaris. In the present study, it was found that distinct salt concentration significantly inhibited the growth of $C$. militaris strains. The colony diameter and biomass of $C$. militaris strains were decreased with the increase of salt concentration (Figure 1 and Supplementary Figure S1). Obviously, C. militaris strain encountered osmotic stress due to the high-salt environment, and the salt tolerance of $C$. militaris cells might be essential for the long-term fermentation. High salinity represents a stressinduced high osmotic perturbation to cells since the excess salt disturbs osmotic potential and leads to metabolic toxicity (43). Analogously, clear growth defects under salinity conditions were also observed in Streptomyces coelicolor, and major changes occurred in the primary and secondary metabolism (Sugars, polyols, amino acids, nucleotides, and their derivatives) (33). 


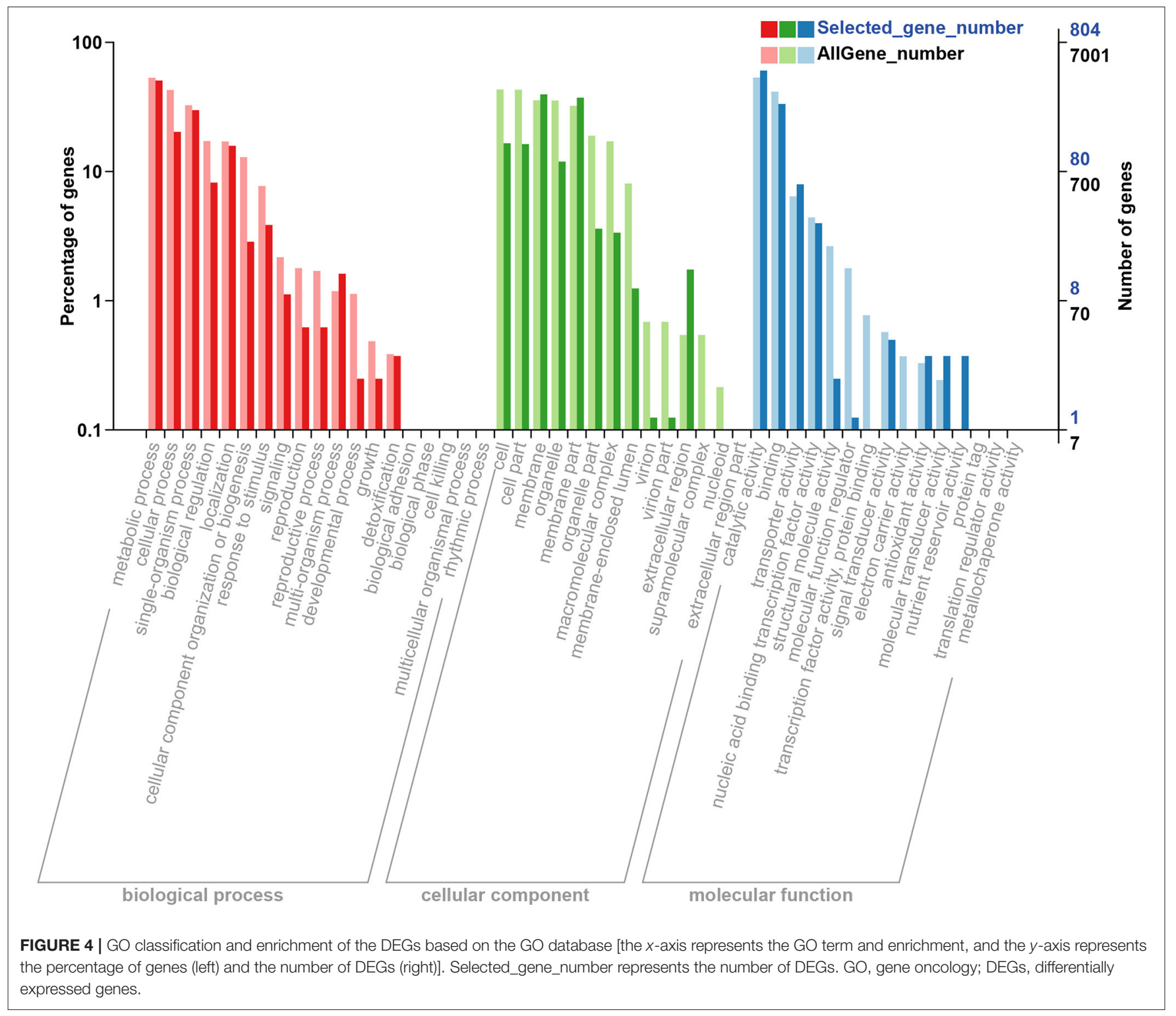

The evolutions of varying morphological, physiological, and molecular mechanisms of adaptation are the main strategies of Halophilic fungi in reflection to osmotic stress conditions (44). Exactly, the morphological responses are meristematic growth, pigmentation, and changes in the cell wall and membrane composition (45). Moreover, the physiological responses consist of intracellular potassium and sodium ion content synthesis, accumulation of organic compatible solutes, and extracellular polysaccharide production. As for the molecular responses, it was reflected in the dynamic changes in gene expression related to physiological responses to enhanced salt concentration (44).

Contrary to the inhibited growth of $C$. militaris strain, the accumulation of cordycepin has a positive correlation with salt concentration after culturing in the PDA plates for 5 days (Figure 2). The yield of cordycepin on the NaCl-7 group was five-fold higher than that without salt supplementary. It was assumed that a high-salt environment might be optimal for cordycepin accumulation, whereas related molecular responses to an increased extracellular saline condition in C. militaris strain have not been identified. To figure out the metabolic pathway from the additive salt to cordycepin, four samples were collected, and transcriptome sequencing was performed based on RPKM metrics. Exposure of $C$. militaris cells to salt treatment results in a substantial transcriptional regulation, and 3,885 genes have altered expressions in three salinity treatment groups relative to the control (Figure 3 ). In the case of the $\mathrm{NaCl}-7$ group, the number of DEGs reached a peak, revealing a greater degree of change in gene expressions in the high salt group than that in the low salt group. In fact, exposure of $C$. militaris cells to the highsalt environment implies threats of specific cation toxicity. So as to adapt to extracellular salinity, there is a transient induction of transcription that activates stress-protective genes $(46,47)$. 


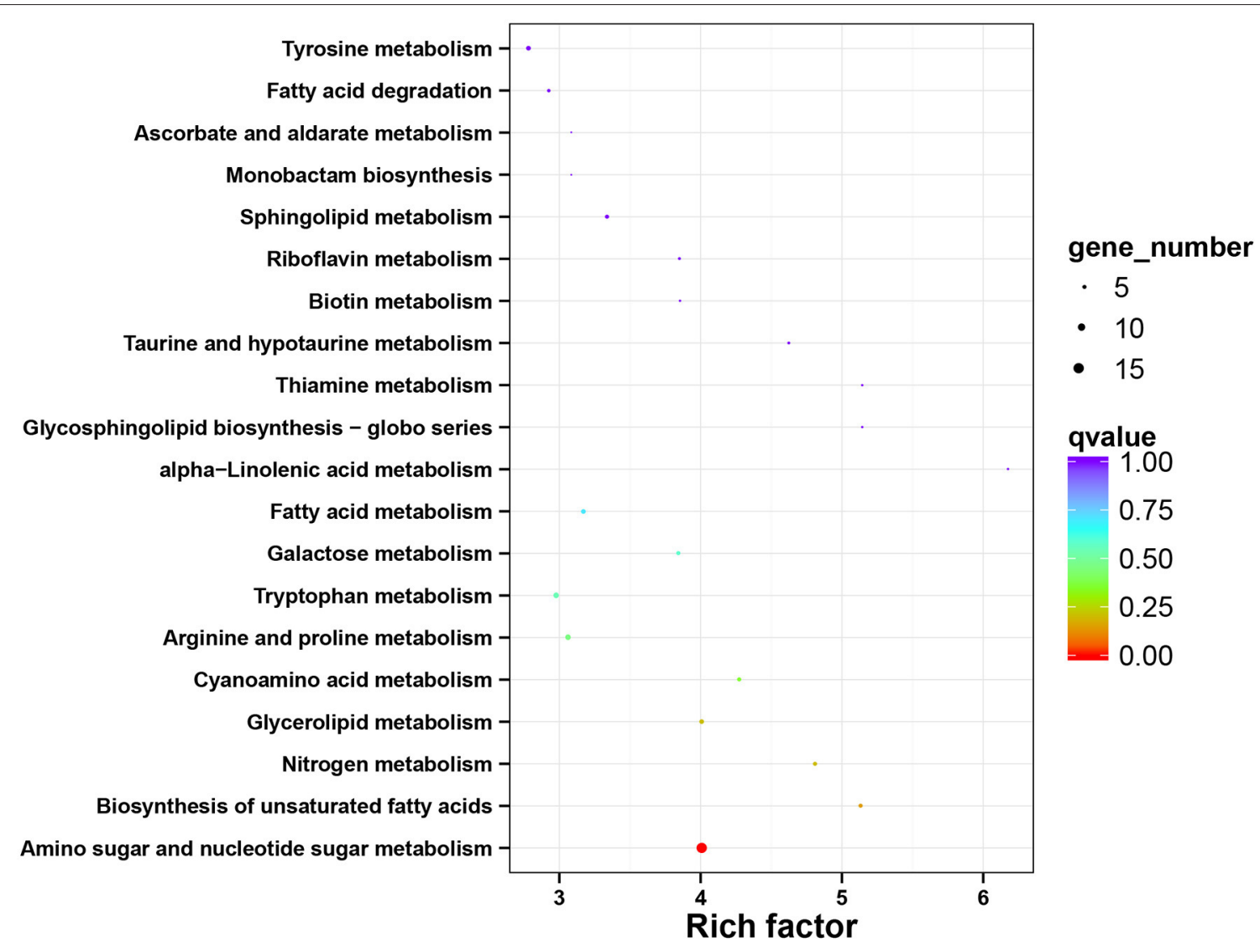

FIGURE 5 | The functional enrichment analysis of DEGs using KEGG annotation. The $x$-axis represents the rich factor, and the $y$-axis represents the pathway name. DEGs, differentially expressed genes; KEGG, kyoto encyclopedia of genes and genomes.

Furthermore, to analyze and classify the DEGs, the sequencing data were uploaded to the GO and KEGG databases (Figures 4, 5). GO classification results of DEGs showed that the salinity treatment may facilitate the metabolic process to produce more metabolites (cordycepin accumulation) and consequently activate membrane transportation (out-pumping of PTN). The cordycepin metabolism and the purine metabolism pathway have been elucidated in detail by comprehensive transcriptome and proteome analysis of $C$. militaris $(21,48)$. It was reported that cordycepin production can be increased by adding L-alanine, and a high transcriptional level of several genes encoding enzymes on the pathway of adenosine synthesis was also observed (8). Similarly, the majority of DEGs classified in "metabolic process" and "catalytic activity" items in this study showed different salt treatments may activate adenosine synthesis-related enzymes. And these enzymes catalyze series reactions related to adenosine synthesis and finally lead to the accumulation of cordycepin. Meanwhile, KEGG classification indicated that the most DEGs significantly enriched in the pathway of "Amino sugar and nucleotide sugar metabolism", "Biosynthesis of unsaturated fatty acids", and "Glycerolipid metabolism". It was illustrated that salt treatment affected the specific energy metabolism and membrane composition changes. Most of the DEGs were relevant to the pathway of "Amino sugar and nucleotide sugar metabolism", which revealed that the addition of salt might increase energy molecule production and further promote the cordycepin accumulation. It is generally accepted that cell membranes are one of the first targets to suffer from injurious effects of stress, leading to impaired cell membrane integrity and functions $(49,50)$. Plenty of studies have documented the modifications of cellular lipid profiles in response to osmotic shock $(51,52)$. In the present study, there were also a large number of DEGs enriched in the pathway of "Biosynthesis of unsaturated fatty acids" and "Glycerolipid metabolism", which was consistent with the abovementioned Halophilic fungi in reflection to salt treatment condition by changing the membrane composition (morphological responses) (45). Additionally, it was reported that glycerolipid metabolism and high osmotic glycerol (HOG) signaling pathway enable micro-organisms to respond to various extracellular stimuli and also to adjust their cellular machinery to change the environments (53). Two isoenzymes of DL-glycerol-3-phosphatase in the glycerolipid metabolism pathway from Saccharomyces cerevisiae facilitate the ability of cells to expose to osmotic stress via the HOG pathway (54). Therefore, numbers of DEGs enriched in the pathway of glycerolipid metabolism in C. militaris strain indicated 


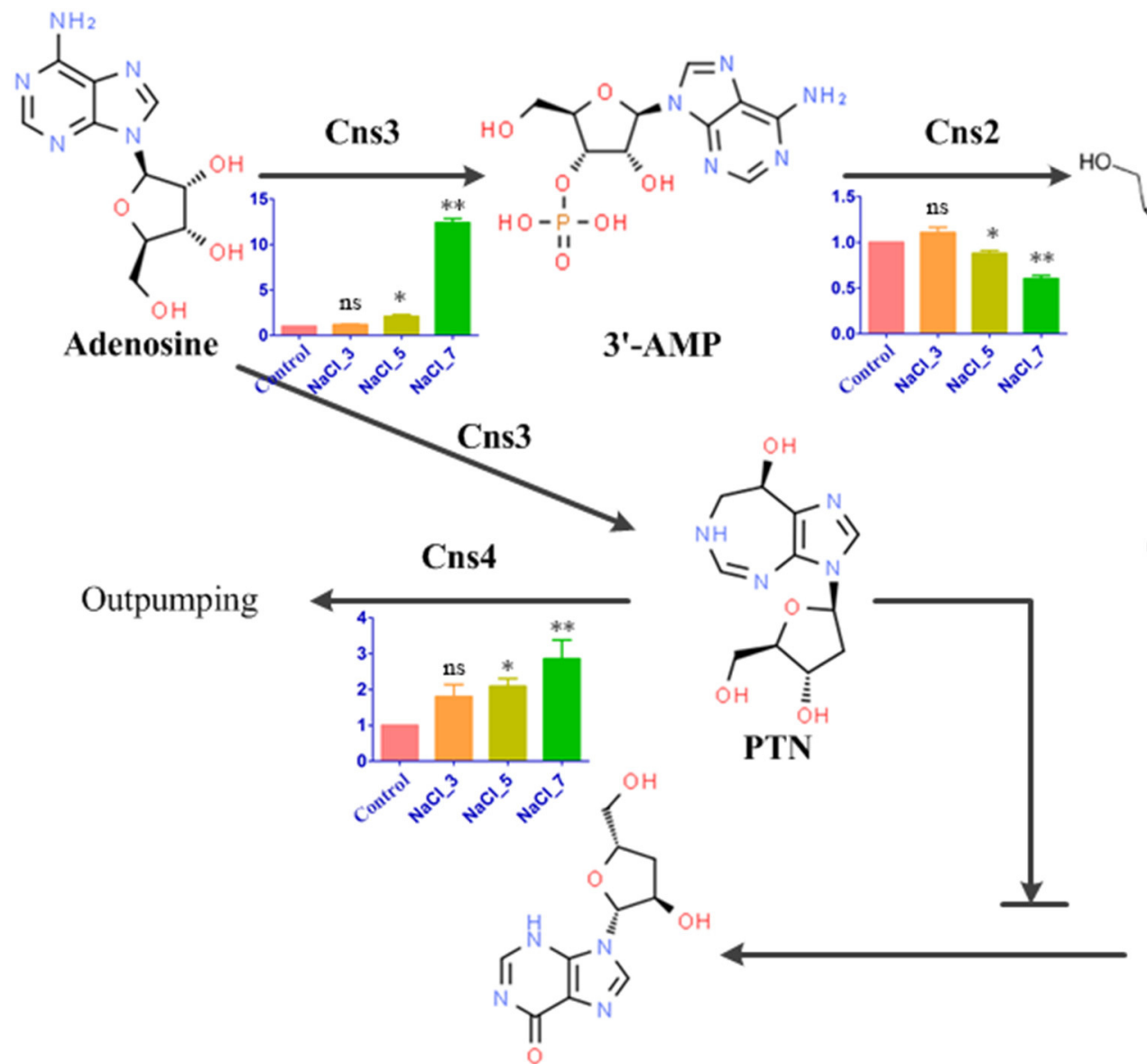

3'-Deoxyinosine<smiles></smiles>

2'-C-3'-dA

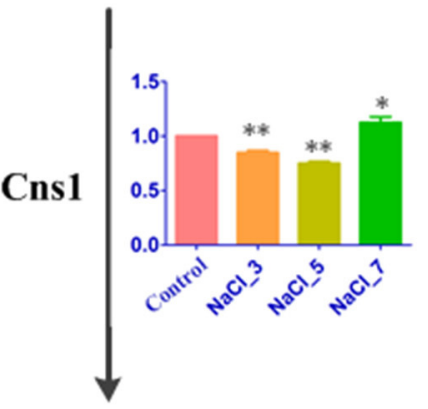

\section{Cordycepin}

FIGURE 6 | Cordycepin biosynthesis pathway in C. militaris and qRT-PCR results. Bar chart with 4 colors represents the relative expression levels of four genes in response to distinct salt treatments. The bars represent the average ( $\pm \mathrm{SE}$ ) of biological repeats. $\mathrm{NaCl}-3,5$, and 7 represent $3 \%, 5 \%$, and $7 \%$ salt concentrations, respectively. Asterisks indicate statistically significant differences between groups (Student's $t$-test): * and ${ }^{\star *}$ indicating significance level was accepted at $P \leq 0.05$ and $P<0.01$, respectively, ns: no significant difference, as compared to control.

the addition of salt may achieve the same effect on that of Saccharomyces cerevisiae.

In the aforementioned context, cordycepin synthesis was mediated by a biosynthetic gene cluster, which consisted of gene Cns1-Cns4 (21). Additionally, PTN, a purine analog with the activity of anticancer and adenosine deaminase inhibition, was noteworthily produced in coupling with cordycepin by Cns3 (55). In the present study, dynamic changes of the transcription level of these four vital genes were observed based on the data obtained by transcriptome analysis. To further confirm the reliability and availability of these four DEGs, a real-time RTPCR experiment was carried out. Overall, these qRT-PCR results showed that the DEGs transcription level was consistent with the result obtained by transcriptome analysis (Figure 6). Genes Cns1, Cns3, and Cns4 were remarkably upregulated under high salt concentration which showed osmotic stress conditions may have beneficial to accumulating the cordycepin and transporting the PTN to the extracellular environment. Cns2 was downregulated under distinct salt treatments (except on the Nacl-3 group), implying that the process of $3^{\prime}$ AMP dephosphorylated to $2^{\prime}$-C$3^{\prime}$-dA by phosphohydrolase activity of Cns 2 was inhibited by salt treatment, and detailed molecular mechanism still needs further experimental validation. In addition, it has been reported that the yield of cordycepin was increased 2.7-fold by overexpression of the Cns1/Cns2 fusion gene (21). Combined with the results in our study, it is worth trying that overexpression of the Cns1/Cns2 or Cns1/Cns2/Cns3 fusion gene via individually transformed the control strain of $C$. militaris under salt treatment. Furthermore, it has been reported that several genes can regulate osmoadaptation, such as salt-tolerant gene HOG and fatty acid metabolism gene delta- 9 fatty acid desaturases $(56,57)$. Thus, it can be expected that the obtaining of industrial strains possesses salt tolerance and high cordycepin production via overexpression of the cordycepin biosynthetic gene and salt-tolerant gene. Interestingly, a significant upregulation of ribonucleotide reductase (RNR, 70.19-fold), $5^{\prime}$-Nucleotidase (NT5E, 63.43-fold), purA (Adenylosuccinate synthase, 8.43-fold), and Adenylate kinase (ADEK, 17.52-fold) gene expression was 
observed upon hypoxanthine treatment, which are downstream genes responsible for cordycepin biosynthesis in Ophiocordyceps sinensis (30). Analogous effects have also been achieved on several other potent growth supplements, such as amino acids, plant hormones, and vitamins. These results indicated both salt treatment and the addition of potent growth supplements can activate the cordycepin biosynthesis pathway leading to improved cordycepin content. Moreover, recent research first reported the transcriptome and proteomics of Cordyceps kyushuensis Kob, which is close relative to C. militaris (58). Similar to C. militaris, a single gene cluster containing $c k 1$ ck4, which can synthesize both cordycepin and PTN, has been identified in Cordyceps kyushuensis Kob using BLAST. Therefore, it is worth exploiting the potential of Cordyceps kyushuensis Kob on the increment of cordycepin production in the near future apart from C. militaris. With the deep analysis of transcriptome and proteomics, more useful information to do contribution to the cosmetic and pharmaceutical industries can be provided by improving the yield of cordycepin.

\section{CONCLUSIONS}

To better unravel the salt response mechanism of C. militaris and its effects on cordycepin content, our research performed a transcriptome analysis of $C$. militaris under the distinct salt treatments. The HPLC analysis depicted high salt treatment was beneficial to enhance cordycepin production. Combined with GO analysis and KEGG pathway enrichment, the upregulated transcriptome level of genes responsible for the biosynthetic pathways of energy generation and lipid metabolism might be the major reason for the accumulation of cordycepin. Finally, mRNA expression analysis of four genes involved in the cordycepin biosynthesis pathway was carried out by RT-PCR, and the results showed that the transcription level of these genes was consistent with that of transcriptome analysis. Taken together, our study provides a global transcriptome characterization of the osmotic stress adaptation process in C. militaris and paves the way for constructing industrial strains that possess salt tolerance and high cordycepin production.

\section{DATA AVAILABILITY STATEMENT}

The datasets presented in this study can be found in online repositories. The names of the repository/repositories and

\section{REFERENCES}

1. Wang L, Zhang WM, Hu B, Chen YQ, Qu LH. Genetic variation of Cordyceps militaris and its allies based on phylogenetic analysis of rDNA ITS sequence data. Fungal Divers. (2008) 31:147-55. Available online at: https://www. fungaldiversity.org/fdp/sfdp/31-11.pdf

2. Shrestha B, Tanaka E, Hyun MW, Han J-G, Kim CS, Jo JW, et al. Coleopteran and lepidopteran hosts of the entomopathogenic genus cordyceps sensu lato. J Mycol. (2016) 2016:1-14. doi: 10.1155/2016/76 48219

3. Das SK, Masuda M, Sakurai A, Sakakibara M. Medicinal uses of the mushroom Cordyceps militaris: current state and prospects. Fitoterapia. (2010) 81:961-8. doi: 10.1016/j.fitote.2010.07.010 accession number(s) can be found below: https://www.ncbi.nlm. nih.gov/, PRJNA770191.

\section{AUTHOR CONTRIBUTIONS}

GL: conceptualization, visualization, and writing-original draft preparation. YZ: conceptualization. XC and YC: project administration. BZ and XL: supervision and funding acquisition. $\mathrm{BH}$ : writing review, editing, and funding acquisition. All authors contributed to the article and approved the submitted version.

\section{FUNDING}

This research was funded by the Natural Science Foundation of Jiangxi Province (Grant Nos. 20202BABL203043, 20202BAB215003, and 20212BAB215005) and Jiangxi Double Thousand Plan Cultivation Program for Distinguished Talents in Scientific and Technological Innovation (jxsq2019201011).

\section{ACKNOWLEDGMENTS}

Thanks to Yayi Tu for the critical review of this manuscript.

\section{SUPPLEMENTARY MATERIAL}

The Supplementary Material for this article can be found online at: https://www.frontiersin.org/articles/10.3389/fnut.2021. 793795/full\#supplementary-material

Figure S1 | Determination of the biomass of the $C$. militaris mycelia under salt treatment. $0,3,5$, and $7 \%$ are equivalent to the control, slight, moderate, and severe salt treatment, respectively.

Figure S2 | HPLC comparison of cordycepin production among the control and three salt treatment groups. 0, 3, 5, and 7\% represent the control, slight, moderate, and severe salt-treated groups, respectively.

Figure S3 | Heatmap of the hierarchical clustering of the DEGs. A, B, and C represent Control vs. $\mathrm{NaCl}-3,-5$, and -7 , respectively, (the $x$-axis represents each compared sample; the $y$-axis represents the DEGs. The coloring indicates the fold change: high, green; low, red). DEGs, differentially expressed genes.

Figure S4 | A comparison of transcriptional level change of genes (Cns1-Cns4) between transcriptome analysis and RT-PCR validation.

Table S1 | qRT-PCR primers used in this study.

Table S2 | The identification of significant DEGs among four treatments (Control vs. NaCl-3, -5, and -7). DEGs, differentially expressed genes.

Table S3 | KEGG pathway enrichment of DEGs. DEGs, differentially expressed genes; KEGG, kyoto encyclopedia of genes and genomes.

4. Zhou X, Gong Z, Su Y, Lin J, Tang K. Cordyceps fungi: natural products, pharmacological functions and developmental products. J Pharm Pharmacol. (2009) 61:279-91. doi: 10.1211/jpp.61.03.0002

5. Dong JZ, Wang SH, Ai XR, Yao L, Sun ZW, Lei C, et al. Composition and characterization of cordyxanthins from Cordyceps militaris fruit bodies. $J$ Funct Foods. (2013) 5:1450-5. doi: 10.1016/j.jff.2013.06.002

6. Cunningham KG, Manson W, Spring FS, Hutchinson SA. Cordycepin. A metabolic product isolated from cultures of cordyceps militaris (Linn) link. Nature. (1950) 166:949-949. doi: 10.1038/166949a0

7. Zhang G, Yin Q, Han T, Zhao Y, Su J, Li M, et al. Purification and antioxidant effect of novel fungal polysaccharides from the stroma of Cordyceps kyushuensis. Ind Crops Prod. (2015) 69:485-91. doi: 10.1016/j.indcrop.2015.03.006 
8. Chen BX, Wei T, Xue LN, Zheng QW, Ye ZW, Zou Y, et al. Transcriptome analysis reveals the flexibility of cordycepin network in cordyceps militaris activated by L-alanine addition. Front Microbiol. (2020) 11:577. doi: $10.3389 /$ fmicb.2020.00577

9. Thananusak R, Laoteng K, Raethong N, Zhang Y, Vongsangnak W. Metabolic responses of carotenoid and cordycepin biosynthetic pathways in cordyceps militaris under light-programming exposure through genome-wide transcriptional analysis. Biology 9:242. doi: 10.3390/biology9090242

10. Tuli HS, Sandhu SS, Sharma AK. Pharmacological and therapeutic potential of cordyceps with special reference to cordycepin. (2014). 4:112. doi: 10.1007/s13205-013-0121-9

11. Holbein S, Wengi A, Decourty L, Freimoser FM, Jacquier A, Dichtl B. Cordycepin interferes with 3 ' end formation in yeast independently of its potential to terminate RNA chain elongation. RNA. (2009) 15:83749. doi: 10.1261/rna.1458909

12. Noh E-M, Kim J-S, Hur H, Park B-H, Song E-K, Han M-K, et al. Cordycepin inhibits IL-1 $\beta$-induced MMP-1 and MMP-3 expression in rheumatoid arthritis synovial fibroblasts. Rheumatology. (2008) 48:458. doi: 10.1093/rheumatology/ken417

13. Pan BS, Lin CY, Huang BM. The effect of cordycepin on steroidogenesis and apoptosis in MA-10 mouse leydig tumor cells. Evid Based Complement Alternat Med. (2011) 2011:750468. doi: 10.1155/2011/750468

14. Tian X, Li Y, Shen Y, Li Q, Wang Q, Feng L. Apoptosis and inhibition of proliferation of cancer cells induced by cordycepin (Review). Oncol Lett. (2015) 10:595-9. doi: 10.3892/ol.2015.3273

15. Fishman P, Bar-Yehuda S, Liang BT, Jacobson KA. Pharmacological and therapeutic effects of A3 adenosine receptor agonists. Drug Discov Today. (2012) 17:359-66. doi: 10.1016/j.drudis.2011.10.007

16. Cho HJ, Cho JY, Rhee MH, Kim HS, Lee HS, Park HJ. Inhibitory effects of cordycepin (3'-deoxyadenosine), a component of Cordyceps militaris, on human platelet aggregation induced by thapsigargin. J Microbiol Biotechnol. (2007) 17:1134-8.

17. Zhou X, Cai G, He Y, Tong G. Separation of cordycepin from Cordyceps militaris fermentation supernatant using preparative HPLC and evaluation of its antibacterial activity as an NAD+-dependent DNA ligase inhibitor. Exp Ther Med. (2016) 12:1812-6. doi: 10.3892/etm.2016.3536

18. Kaczka EA, Trenner NR, Arison B, Walker RW, Folkers K. Identification of cordycepin, a metabolite of Cordyceps militaris, as 3'-deoxyadenosine. Biochem Biophys Res Commun. (1964) 14:456-7. doi: 10.1016/0006-291X(64)90086-5

19. Hanessian S, DeJongh D, McCloskey JA. Further evidence on the structure of cordycepin. Biochimica et Biophysica Acta (BBA)-General Subjects. (1966) 117:480-2. doi: 10.1016/0304-4165(66)90101-2

20. Zheng P, Xia Y, Xiao G, Xiong C, Hu X, Zhang S, et al. Genome sequence of the insect pathogenic fungus Cordyceps militaris, a valued traditional chinese medicine. Genome Biol. (2011) 12:R116. doi: 10.1186/gb-2011-12-11-r116

21. Xia Y, Luo F, Shang Y, Chen P, Lu Y, Wang C. Fungal cordycepin biosynthesis is coupled with the production of the safeguard molecule pentostatin. Cell Chem Biol. (2017) 24:1479-89 e1474. doi: 10.1016/j.chembiol.2017.09.001

22. Tuli HS, Sharma AK, Sandhu SS, Kashyap D. Cordycepin: A bioactive metabolite with therapeutic potential. Life Sci. (2013) 93:863-9. doi: 10.1016/j.lfs.2013.09.030

23. Wu P, Wan D, Xu G, Wang G, Ma H, Wang T, et al. An unusual protectorprotégé strategy for the biosynthesis of purine nucleoside antibiotics. Cell Chem Biol. (2017) 24:171-81. doi: 10.1016/j.chembiol.2016.12.012

24. Kunhorm P, Chaicharoenaudomrung N, Noisa P. Enrichment of cordycepin for cosmeceutical applications: culture systems and strategies. Appl Microbiol Biotechnol. (2019) 103:1681-91. doi: 10.1007/s00253-019-09623-3

25. Dong JZ, Lei C, Ai XR, Wang Y. Selenium enrichment on cordyceps militaris link and analysis on its main active components. Appl Biochem Biotechnol. (2012) 166:1215-24. doi: 10.1007/s12010-011-9506-6

26. Fan D-d, Wang W, Zhong J-J. Enhancement of cordycepin production in submerged cultures of Cordyceps militaris by addition of ferrous sulfate. Biochem Eng J. (2012) 60:30-5. doi: 10.1016/j.bej.2011.09.014

27. Lin L-T, Lai Y-J, Wu S-C, Hsu W-H, Tai C-J. Optimal conditions for cordycepin production in surface liquid-cultured Cordyceps militaris treated with porcine liver extracts for suppression of oral cancer. J Food Drug Anal. (2018) 26:135-44. doi: 10.1016/j.jfda.2016.11.021
28. Tang J, Qian Z, Wu H. Enhancing cordycepin production in liquid static cultivation of Cordyceps militaris by adding vegetable oils as the secondary carbon source. Bioresour Technol. (2018) 268:60-7. doi: 10.1016/j.biortech.2018.07.128

29. Lee SK, Lee JH, Kim HR, Chun Y, Lee JH, Yoo HY, et al. Improved cordycepin production by cordyceps militaris KYL05 using casein hydrolysate in submerged conditions. Biomolecules. (2019) 9:461. doi: 10.3390/biom90 90461

30. Kaushik V, Singh A, Arya A, Sindhu SC, Sindhu A, Singh A. Enhanced production of cordycepin in Ophiocordyceps sinensis using growth supplements under submerged conditions. Biotechnol Rep. (2020) 28:e00557. doi: 10.1016/j.btre.2020.e00557

31. Cai X, Jin JY, Zhang B, Liu ZQ, Zheng YG. Improvement of cordycepin production by an isolated Paecilomyces hepiali mutant from combinatorial mutation breeding and medium screening. Bioprocess Biosyst Eng. (2021) 44:2387-98. doi: 10.1007/s00449-021-02611-w

32. Mishra AK, Singh SS. Protection against salt toxicity in Azolla pinnataAnabaena azollae symbiotic association by using combined-N sources. Acta Biol Hung. (2006) 57:355-65. doi: 10.1556/ABiol.57.2006.3.9

33. Kol S, Merlo ME, Scheltema RA, de Vries M, Vonk RJ, Kikkert $\mathrm{NA}$, et al. Metabolomic characterization of the salt stress response in Streptomyces coelicolor. Appl Environ Microbiol. (2010) 76:257481. doi: 10.1128/AEM.01992-09

34. He B, Ma L, Hu Z, Li H, Ai M, Long C, et al. Deep sequencing analysis of transcriptomes in Aspergillus oryzae in response to salinity stress. Appl Microbiol Biotechnol. (2018) 102:897-906. doi: 10.1007/s00253-017-8603-Z

35. He B, Hu Z, Ma L, Li H, Ai M, Han J, et al. Transcriptome analysis of different growth stages of Aspergillus oryzae reveals dynamic changes of distinct classes of genes during growth. BMC Microbiol. (2018) 18:12. doi: 10.1186/s12866-018-1158-z

36. Langmead B, Salzberg SL. Fast gapped-read alignment with Bowtie 2. Nat Methods. (2012) 9:357-9. doi: 10.1038/nmeth.1923

37. Kim D, Salzberg SL. TopHat-Fusion: an algorithm for discovery of novel fusion transcripts. Genome Biol. (2011) 12:R72. doi: 10.1186/gb-2011-12-8-r72

38. Mortazavi A, Williams BA, McCue K, Schaeffer L, Wold B. Mapping and quantifying mammalian transcriptomes by RNA-Seq. Nat Methods. (2008) 5:621-8. doi: 10.1038/nmeth.1226

39. Dewey CN, Bo LJBB. RSEM: accurate transcript quantification from RNA-Seq data with or without a reference. Genome. (2011) 12:3233. doi: 10.1186/1471-2105-12-323

40. Love MI, Huber W, Anders S. Moderated estimation of fold change and dispersion for RNA-Seq data with DESeq2. Genome Biol. (2014) 15:550. doi: 10.1186/s13059-014-0550-8

41. Ashburner M, Ball CA, Blake JA, Botstein D, Butler H, Cherry JM, et al. Gene Ontology: tool for the unification of biology. Nat Genet. (2000) 25:259. doi: $10.1038 / 75556$

42. Kanehisa M, Araki M, Goto S, Hattori M, Hirakawa M, Itoh M, et al. KEGG for linking genomes to life and the environment. Nucleic Acids Res. (2008) 36(Database issue):D480-4. doi: 10.1093/nar/gkm882

43. Taymaz-Nikerel H, Cankorur-Cetinkaya A, Kirdar B. GenomeWide Transcriptional response of Saccharomyces cerevisiae to stress-induced perturbations. Front Bioeng Biotechnol. (2016) 4:17. doi: 10.3389/fbioe.2016.00017

44. Musa H, Kasim FH, Nagoor Gunny AA, Gopinath SCB. Salt-adapted moulds and yeasts: Potentials in industrial and environmental biotechnology. Process Biochem. (2018) 69:33-44. doi: 10.1016/j.procbio.2018.03.026

45. Zajc J, Zalar P, Plemenitaš A, Gunde-Cimerman N. The mycobiota of the salterns. In: Raghukumar C, editor. Biology of Marine Fungi. Berlin, Heidelberg: Springer (2012). p. 133-58. doi: 10.1007/978-3-642-23342-5_7

46. López-Maury L, Marguerat S, Bähler J. Tuning gene expression to changing environments: from rapid responses to evolutionary adaptation. Nat Rev Genet. (2008) 9:583-93. doi: 10.1038/nrg2398

47. Martínez-Montañés F, Pascual-Ahuir A, Proft M. Toward a genomic view of the gene expression program regulated by osmostress in yeast. Omics. (2010) 14:619-27. doi: 10.1089/omi.2010.0046

48. Yin $\mathrm{Y}, \mathrm{Yu} \mathrm{G}$, Chen $\mathrm{Y}$, Jiang $\mathrm{S}$, Wang $\mathrm{M}$, Jin $\mathrm{Y}$, et al Genome-wide transcriptome and proteome analysis on different 
developmental stages of Cordyceps militaris. PLoS ONE. (2012) 7:e51853. doi: 10.1371/journal.pone.0051853

49. Bajji M, Kinet J-M, Lutts $\mathrm{S}$. The use of the electrolyte leakage method for assessing cell membrane stability as a water stress tolerance test in durum wheat. Plant Growth Regul. (2002) 36:61-70. doi: 10.1023/A:10147327 14549

50. Izzo V, Bravo-San Pedro JM, Sica V, Kroemer G, Galluzzi L. Mitochondrial Permeability Transition: New Findings and Persisting Uncertainties. Trends Cell Biol. (2016) 26:655-67. doi: 10.1016/j.tcb.2016.04.006

51. Mutnuri S, Vasudevan N, Kastner M, Heipieper HJ. Changes in fatty acid composition of Chromohalobacter israelensis with varying salt concentrations. Curr Microbiol. (2005) 50:1514. doi: 10.1007/s00284-004-4396-2

52. Srivastava A, Singh SS, Mishra AK. Modulation in fatty acid composition influences salinity stress tolerance in Frankia strains. Ann Microbiol. (2014) 64:1315-23. doi: 10.1007/s13213-013-0775-x

53. Plemenitaš A, Gunde-Cimerman N. Cellular responses in the halophilic black yeast hortaea werneckii to high environmental salinity. In: GundeCimerman N, Oren A, Plemenitaš A, editors. Adaptation to Life at High Salt Concentrations in Archaea, Bacteria, and Eukarya. Netherlands:Springer. (2005). p. 453-70. doi: 10.1007/1-4020-3633-7_29

54. Norbeck J, Påhlman A-K, Akhtar N, Blomberg A, Adler L. Purification and Characterization of Two Isoenzymes of DL-Glycerol-3-phosphatase from Saccharomyces cerevisiae: identification of the corresponding GPP1 and GPP2 genes and evidence for osmotic regulation of Gpp2p expression by the osmosensing mitogen-activated protein kinase signal transduction pathway*. J Biol Chem. (1996) 271:13875-81. doi: 10.1074/jbc.271.23. 13875

55. Kane BJ, Kuhn JG, Roush MK. Pentostatin: an adenosine deaminase inhibitor for the treatment of hairy cell leukemia. Ann Pharmacother. (1992) 26:93947. doi: $10.1177 / 106002809202600718$
56. Li H, Ma L, Hu Z, Tu Y, Jiang C, Wu Q, et al. Heterologous expression of AoD9D enhances salt tolerance with increased accumulation of unsaturated fatty acid in transgenic Saccharomyces cerevisiae. J Ind Microbiol Biotechnol. (2019) 46:231-9. doi: 10.1007/s10295-018-02123-9

57. Kuo CY, Chen SA, Hsueh YP. The High Osmolarity Glycerol (HOG) pathway functions in osmosensing, trap morphogenesis and conidiation of the nematode-trapping fungus Arthrobotrys oligospora. J Fungi. (2020) 6:191. doi: 10.3390/jof6040191

58. Zhao X, Zhang G, Li C, Ling J. Cordycepin and pentostatin biosynthesis gene identified through transcriptome and proteomics analysis of Cordyceps kyushuensis Kob. Microbiol Res. (2019) 218:12-21. doi: 10.1016/j.micres.2018.09.005

Conflict of Interest: The authors declare that the research was conducted in the absence of any commercial or financial relationships that could be construed as a potential conflict of interest.

Publisher's Note: All claims expressed in this article are solely those of the authors and do not necessarily represent those of their affiliated organizations, or those of the publisher, the editors and the reviewers. Any product that may be evaluated in this article, or claim that may be made by its manufacturer, is not guaranteed or endorsed by the publisher.

Copyright $\odot 2021 \mathrm{Lv}, \mathrm{Zhu}$, Cheng, Cao, Zeng, Liu and He. This is an open-access article distributed under the terms of the Creative Commons Attribution License (CC $B Y)$. The use, distribution or reproduction in other forums is permitted, provided the original author(s) and the copyright owner(s) are credited and that the original publication in this journal is cited, in accordance with accepted academic practice. No use, distribution or reproduction is permitted which does not comply with these terms. 